\title{
Simplified Link Reliability Enhancement Schemes for Wireless Networks
}

\author{
Boon Hoi Lim ${ }^{1}$, Chong Eng Tan ${ }^{2}$ \\ Department of Computer Systems and Communications Technologies \\ Faculty of Computer Science and Information Technology \\ Universiti Malaysia Sarawak \\ 94300 Kota Samarahan, Malaysia. \\ boonhoi83@gmail.com ${ }^{1}$, cetan@fit.unimas.my ${ }^{2}$
}

\begin{abstract}
Today's Internet uses the IP protocol suite that was primarily designed for the transport of data and provides best-effort data delivery. Thus, it does not guarantee the quality of voice transmission that is highly sensitive to error and delay. The situation is even more severe in wireless networks which experience even more distortion and attenuation. Therefore, enhancing the voice traffic quality in the wireless network is essentials for users to experience the lowest erroneous voice traffic. The objective of this paper is to propose an enhanced link reliability scheme and a low level frame prioritize scheme where they are able to improve the QoS in voice traffic especially in wireless networks. Each scheme utilized the wireless environment to tackle the time sensitive traffic. The two schemes have been proven to be able to performance well in enhancing voice quality under specified circumstances via network simulations.
\end{abstract}

\section{Introduction}

Voice over Internet Protocol (VoIP) is a technology that allows people to make phone calls through the public Internet rather than the traditional Publish Switched Telephone Network (PSTN). VoIP is a common term refers to different protocols that are used to transport real-time voice and the necessary signaling by means of the Internet Protocol (IP) [1]. With increasing demands in mobility and flexibility, the market for wireless network is evolving to incorporate high-speed Internet access and advance multimedia transmission.

Today's Internet uses the IP protocol suite that was designed for the transport of data packet and can only provides best-effort data delivery. Thus, network in this nature does not guarantee a minimum packet loss rate and minimum delay of packet transmission required for Internet Telephony where additional QoS schemes may need to be applied in order to prioritize time sensitive transmission especially for real time applications. Due to the realtime requirement for interactive voice transmission, it is impractical for the receiver to request for retransmitting the lost packets. The voice packets that do not arrive before their playout time expires are considered lost and cannot be played when they received at a later time [4]. This problem is more severe when come to transmission over the wireless networks with very rough channel condition. Therefore, a less complex and yet easy to implement QoS scheme is essential for the transmission of time sensitive data over the wireless networks.

In most of the wireless environment, Forward Error Correction (FEC) mechanism is used to quickly recovery the loss packets especially in fullduplex environment. FEC mechanism has its own limitations where when the network loss rate is high, the packet received from the IP network will still be corrupted and the data is unable to be recovered via the FEC scheme [8]. The inefficiency caused by the FEC scheme can indirectly influence the QoS of multimedia traffic transmissions. Even though the popular FEC scheme is widely used in wireless network, it will still cause variation in bandwidth and delay. In other words, it causes congestion loss due to buffer overflow or erroneous loss in wireless link [4] [5].

There is plenty of point-to-point bandwidth available in wireless networks especially between the end nodes and the access point. The data-rate available in wireless network i.e., WLAN (IEEE802.11a/g/n) is more than sufficient to providing the required bandwidth for Internet access packets. Thus, the research drive for this paper is to improve and simplify the QoS mechanism for VoIP especially in wireless link by making use of the excess bandwidth available in the wireless networks. The proposed schemes fully utilize this advantage of highly available bandwidth in the wireless section to improve the VoIP QoS over the Internet. This is because in most cases, the Internet gateway bandwidth is only a small fraction of the bandwidth that the wireless networks offer. Hence, it is practical 